\title{
UVEITIS FOLLOWING ANTI-SNAKE VENOM THERAPY
}

NAYAK S. G. (1), SATISH R. (1), NITYANANDAM S. (2), THOMAS R. K. (2)

(1) Department of Nephrology, St. John's Medical College Hospital, Bangalore, Karnataka State, India; (2) Department of Ophthalmology, St. John's Medical College Hospital, Bangalore, Karnataka State, India.

ABSTRACT: Snakebite is a common medical emergency in the tropics, causing multisystemic involvement. Ophthalmic manifestations after snakebite have included ptosis and ophthalmoplegia. Uveitis as an immunological complication following therapy with anti-snake venom (ASV) serum has been rarely reported in literature. We reported two patients who developed uveitis and acute renal failure following snakebite treatment with ASV serum. Both patients recovered uneventfully with appropriate therapy.

KEY WORDS: snakebite, uveitis, anti-snake venom, ptosis, ophthalmoplegia.

CONFLICTS OF INTEREST: There is no conflict.

\section{CORRESPONDENCE TO:}

SHOBHANA G. NAYAK, Department of Nephrology, St. John's Medical College Hospital, Sarjapur Road, Bangalore, 560034, Karnataka State, India. Phone: 9180 2206 5301. Fax: 91802563 3844. Email: nayak shobhana@rediffmail.com. 


\section{INTRODUCTION}

Ocular manifestations resulting from snake envenomation are myriad and vary with the nature of the toxin. Elapidae (cobras and kraits) venoms are mainly neurotoxic and may cause neuro-ophthalmologic manifestations. These include diplopia, poor visual acuity, ophthalmoplegia and ptosis. Viperidae venoms are hematotoxic and may lead to subconjunctival hemorrhage, hyphema, retinal and vitreous hemorrhages.

Uveitis associated with snakebites is rare, and only one case has been reported in literature (1). We reported two patients presenting viper envenomation who developed bilateral uveitis after receiving anti-snakevenom (ASV) therapy.

\section{CASE 1}

A 20-year-old man sought treatment for oliguria and epistaxis following viper envenomation two days prior to admission at St. John's Medical College Hospital, Bangalore, Karnataka State, India. The patient had received anti-snake venom sera at a primary treatment center.

Systemically, the patient was conscious, alert and stable. Hematological evaluation revealed a marked reduction in platelet count $\left(5,000 / \mathrm{mm}^{3}\right)$ and low hemoglobin levels $(7.5 \mathrm{mg} / \mathrm{dl})$. Prothrombin time (patient, 20.4s; control, 17s) and activated prothrombin time (APTT: patient, 43s; control, 34s) were prolonged. Renal function tests were abnormal: blood urea (BU), 303mg/dl; serum creatinine, $12.2 \mathrm{mg} / \mathrm{dl}$. Urine analysis was normal. He was treated with hemodialysis with multiple transfusions and antisnake venom sera. The patient sought an ophthalmology consult 15 days after the snakebite due to rapidly, progressive, painless loss of vision in both eyes. Ocular examination revealed visual acuity of $6 / 18$; <N36 both eyes, which deteriorated to $4 / 60$ over the next 24 hours. There was bilateral subconjunctival hemorrhage. Pupils were sluggishly reactive to light. Slit lamp examination showed 2+ cells and flare, as well as 3+ retrolental cells. Fundus view was hazy due to vitritis, hyperemic optic disc and tortuous retinal blood vessels. B-scan ultrasound showed a few vitreous echoes, and the rest of the posterior segment was normal. Intravenous methyl prednisolone (IV MP) was administered at the dose of $1 \mathrm{~g} /$ day for 3 days. Thereafter, the patient received oral prednisolone at the dose of 40mg/day, which was tapered over 4 weeks. At the beginning of IV MP injections, renal functions were grossly abnormal although the hematological parameters were normal. 
Visual acuity improved to 6/9; N6 both eyes, with marked reduction of vitritis on day 3 of IV MP treatment. There was significant improvement in renal functions and serum creatinine increased from $11.5 \mathrm{mg} / \mathrm{dl}$ to $4.4 \mathrm{mg} / \mathrm{dl}$ over the next 7 days. After 2 months of follow up, visual acuity was 6/6, N6 presented normal color vision and no evidence of active uveitis. Renal functions at follow up were: $\mathrm{BU}, 45 \mathrm{mg} / \mathrm{dl}$, serum creatinine, $1.5 \mathrm{mg} / \mathrm{dl}$.

\section{CASE 2}

A 60-year-old man was admitted to St. John's Medical College Hospital with a history of snakebite by Russel's viper on the dorsum of his left foot 10 days before. He had received anti-snake venom serum at a local governmental center before being referred to our center due to worsening renal functions.

His hematological and coagulation profiles were normal. Renal functions were abnormal; blood urea concentration was $210 \mathrm{mg} \%$ and serum creatinine concentration was $7.5 \mathrm{mg} \%$. He showed normal serum electrolytes and blood gas analysis. The patient was non-oliguric at presentation and could be managed conservatively for renal failure. One day after admission, he complained of redness and watering in both eyes. Ocular examination showed ciliary congestion and lid edema. Extraocular movements were normal. Vision was diminished to 6/18 in both eyes. Slit lamp examination showed $2+$ cells and flare. Pupillary reactions were sluggish in both eyes. The intraocular pressure, measured by indentation tonometry, was normal in both eyes. Fundus examination was normal. The patient was treated with IV MP followed by a short course of oral steroids, $60 \mathrm{mg} /$ day, for 2 weeks.

The uveitis responded well to treatment. His renal functions also recovered spontaneously and did not require dialysis. He was discharged presenting $2.1 \mathrm{mg} \%$ serum creatinine. One month later, his visual acuity was $6 / 6$ and serum creatinine concentration was $1.2 \mathrm{mg} / \mathrm{dl}$.

\section{DISCUSSION}

Systemic manifestations of snakebite depend on the various polypeptide toxins present in the venom. Neurotoxic manifestations can appear as early as 3 minutes after the bite but may be delayed for up to 19 hours (5). Ocular manifestations resulting from snakebite have been uncommonly reported in literature. Subconjunctival hemorrhage, hyphema, retinal and vitreous hemorrhages are well- 
known complications of viper envenomation. Other rare complications described included ptosis, ophthalmoplegia, central retinal artery occlusion, optic neuritis, optic atrophy, cortical blindness, and angle closure glaucoma (1, 4, 6-8). Ptosis and ophthalmoplegia may be the only manifestations following elapid bites. Cobra venom can lead to blindness due to retinal cells damage causing bilateral optic neuritis (2, $6)$.

Uveitis following snakebite has been reported earlier (3). It could be part of serum sickness like a response to horse ASV serum (3).

Both our patients developed severe uveitis 10-15 days following snakebite. The late onset of uveitis excludes the direct effects of the snake toxins on its pathogenesis. Vitritis was probably an immune response to ASV sera. The response to systemic steroids also favors an immune response as the pathogenetic mechanism for this uveitis.

In conclusion, uveitis due to snakebites, although rare, can be very severe leading to vision threatening situations. Physicians and ophthalmologists should always consider the possibility of severe uveitis so that timely and appropriate treatment can be instituted. Immune reactions to equine serum are rare but can lead to increased morbidity, which, if recognized early, will respond to therapy.

\section{REFERENCES}

1 ARI AB. Patient with purely extra-ocular manifestations from pit viper snakebite. Mil. Med., 2001, 166, 667-69.

2 BERGER RR., BROOK S. Cobrabite: Ophthalmic manifestations. Herafuah, 1993, 125, 265-6 (in Hebrew).

3 BUTTES GP., AYAN N., CAMI G. Uveitis after snakebite. Arch. Pediatr., 1996, 3, 832-3.

4 DHALIWAL U. Cortical blindness: an unusual sequela of snakebite. Indian J. Ophthalmol., 1999, 47, 191-2.

5 MITRAKUL C., DHAMKRONG-AT A., FUTRAKAL P., THISYAKORN C., VONGSRISART K., VARAVITHYA C., PHANCHAROEN S. Clinical features of neurotoxic snakebite and response to antivenom in 47 children. Am. J. Trop. Med. Hyg., 1984, 33, 1258-66. 
S. G. Nayak et al. UVEITIS FOLLOWING ANTI-SNAKE VENOM THERAPY. J. Venom. Anim. Toxins incl. Trop. Dis., 2007, 13, 1, p. 134

6 SANGHAVI NG., AMIN SK., NAIK RS. Bilateral optic neuritis following snakebite. J. Assoc. Physicians India, 1982, 30, 117-8.

7 SCHWERSENSKI J., BEATTY DW. Unusual features in a case of snakebite, presumably due to a Cape Cobra (Naja nigricollis). S. Afr. Med. J., 1982, 61, 597-8.

8 SRINIVASAN R., KALIAPERUMAL S., DUTTA TK. Bilateral angle closure glaucoma following snakebite. J. Assoc. Physicians India, 2005, 53, 46-8. 